\title{
ERRATUM \\ Kondo Effect in Single Crystals of Dilute Y-Ce Alloys
}

\author{
T. Sugawara and S. Yoshida
}

Figure 5 of this paper that appears in Journal of Low Temperature Physics 4 , 657 (1971) should be amended as follows: The ordinate $\Delta C / T\left(\mathrm{mj} / \mathrm{gr} \mathrm{K}^{2}\right)$ should be read as $\Delta C / c T\left(10^{-2} \mathrm{~J} /\right.$ mole $\cdot$ at $\left.\% \cdot \mathrm{K}^{2}\right)$. 